\title{
In vitro and in vivo evaluation of the safety and efficacy of a novel liquid fiducial marker for image-guided radiotherapy
}

\author{
LIANG-CHAO SUN ${ }^{1,2}$, YI SU ${ }^{3}$, XING-CHEN DING ${ }^{2}$, DONG-SHUI XU ${ }^{2}$, CHENG-MING LI $^{2}$, \\ LU WANG $^{2}$, WAN-LONG LI ${ }^{2}$, XIN-DONG SUN ${ }^{2}$, JIN-MING YU ${ }^{2}$ and XUE MENG ${ }^{2}$ \\ ${ }^{1}$ Department of Radiation Oncology and Key Laboratory of Cancer Prevention and Therapy, \\ Tianjin Medical University Cancer Institute and Hospital, National Clinical Research Center for Cancer, \\ Tianjin's Clinical Research Center for Cancer, Tianjin, Shandong 300060; ${ }^{2}$ Department of Radiation Oncology, \\ Shandong Cancer Hospital and Institute, Shandong First Medical University and Shandong Academy of Medical Sciences, \\ Jinan, Shandong 250117; ${ }^{3}$ Department of Radiotherapy, The Affiliated Yantai Yuhuangding Hospital of \\ Qingdao University Institution, Yantai, Shandong 264001, P.R. China
}

Received May 7, 2019; Accepted March 26, 2020

DOI: $10.3892 / 01.2020 .11591$

\begin{abstract}
The true extent of a tumor is difficult to visualize, during radiotherapy, using current modalities. In the present study, the safety and feasibility of a mixture of N-butyl cyanoacrylate and lipiodol (NBCA/Lip) was evaluated in order to investigate the optimal combination for application as a fiducial marker for radiotherapy. Four combinations of NBCA/Lip injection (1:1-0.1, 1:1-0.15, 1:3-0.1 and 1:3-0.15 ml) were injected into the subcutaneous tissue of BALB/c mice. The changes in gross histopathology, body weight, skin score, marker volume, neutrophil and macrophage counts were observed to analyze the effects of the different mixing ratios and injection volumes, in order to identify the best combination. Evaluation according to the International Organization for Standardization criteria was further conducted in order to test the biocompatibility of the mixture, including an acute systematic assay with mice, cytotoxicity with L929 fibroblasts and delayed-type hypersensitivity tests with guinea pigs and an intradermal test with rabbits. The results revealed that at the seventh week, 42 markers $(42 / 48 ; 87.5 \%)$ were still visible using computed tomography (CT) imaging. No serious adverse effects were observed throughout the study period; however, the combination of 1:1-0.1 ml had the lowest body weight and worst skin score. A review of the histopathological reaction to NBCA/Lip revealed a combination of acute inflammation, chronic inflammation, granulation tissue, foreign-body
\end{abstract}

Correspondence to: Dr Xue Meng, Department of Radiation Oncology, Shandong Cancer Hospital and Institute, Shandong First Medical University and Shandong Academy of Medical Sciences, 440 Jiyan Road, Jinan, Shandong 250117, P.R. China

E-mail: mengxue5409@163.com

Key words: liquid fiducial marker, n-butyl cyanoacrylate, image-guided radiotherapy, biocompatibility, esophageal cancer reaction and fibrous capsule formation. The 1:1 NBCA combination ratio resulted in the most intense tissue repair reaction and a slower degradation rate of markers. In general, the combination of 1:3-0.15 $\mathrm{ml}$ had a better fusion with local tissue, maintained a stable imaging nodule on CT images for 7 weeks and the final biocompatibility test demonstrated its safety. Overall, the findings of the present study demonstrated NBCA/Lip as a safe and feasible fiducial marker, when using the $1: 3-0.15 \mathrm{ml}$ combination.

\section{Introduction}

Radiotherapy serves an increasingly important role in the multimodality treatment of esophageal cancer $(1,2)$. However, esophageal cancer presents difficulties for precision radiotherapy, such as uncertainties with target delineation and intra-/interfractional tumor position variations (2). One important factor is that the true extent of a tumor is difficult to visualize using computed tomographic (CT) images, which is crucial for the planning of radiotherapy despite the assistance of other current medical examinations, such as esophageal barium meal, MRI(Magnetic Resonance Imaging) and PET-CT(Positron Emission Tomography-Computed Tomography) (3). Endoscopy is used as the standard for determining mucosal tumor extensions in the craniocaudal direction and the endoscopic ultrasonography (EUS)-guided placement of fiducial markers is a novel method prior to targeted radiation therapy, wherein fiducial markers are implanted into the target lesion margin to target and track the location of the tumor in real time. This may provide higher delineation accuracy and the possibility of daily image-guided treatment set-up verification for image-guided radiotherapy (IGRT) (4,5). Previous studies have focused on the use of metal clips, including silver, gold and titanics; however, a high rate of hemorrhage and perforation, risk of chyme rub, esophageal peristalsis and a short residence time limit further application of such solid markers in clinical use (2,5-9). Liquid radiopaque materials have several advantages over metal clips for IGRT, including 
strong attachment to the subcutaneous tissue, controllable density, smaller and controllable size and that these materials can be metabolized (10-12). The use of lipiodol (Lip) has been studied in bladder cancer and lung cancer, wherein Lip is readily identifiable using radiographic and $\mathrm{CT}$ imaging without major adverse side effects. However, the disadvantages of using lipiodol alone include extravasations and the difficulty in producing a consistent marker size (13).

The combination of N-butyl cyanoacrylate (NBCA) and Lip as an embolization treatment has been widely used (14). The use of NBCA as a fiducial marker in IGRT has the potential to overcome the aforementioned shortcomings of IGRT. Dilution of NBCA using lipiodol for intravascular embolization treatment has been shown to affect the duration of vascular occlusion, radiopacity and the polymerization speed of the fiducial marker, which could lead to the catheter becoming sticky or delays embolization and activates cell infiltration $(14,15)$. However, the appropriate mixing ratio and injection dosage of NBCA/Lip as fiducial markers is unknown and the safety and feasibility of this combination remains unclear, highlighting the need for further research prior to its use in clinical practice.

In the present study, in order to identify the most appropriate application of NBCA/Lip as fiducial markers, four different mixing ratios and injection volumes were injected into subcutaneous mouse tissue, followed by quantitative macroscopic and histopathological comparisons to determine the safety of these combinations. Finally, the most appropriate combination was selected and the biocompatibility was further assessed based on the International Organization for Standardization (ISO) (16-18).

\section{Materials and methods}

Reagents and animals. The sterile ready-to-inject NBCA liquid (Histoacryl; B. Braun Melsungen AG) was diluted using lipiodol, using pipettes and 22-gauge needles. A total of 224 BALB/c 6-week-old male mice (Hua Fukang Bioscience, Ltd.), weighing 16-22 g; 30 3-month-old male Hartley Albino guinea pigs (Hua Fukang Bioscience, Ltd.), weighing 300-400 g; and two two-month-old male New Zealand white rabbits (Hua Fukang Bioscience, Ltd.), weighing 2-2.5 kg were allowed free access to food and water throughout the experiments. The housing conditions were as follows: $24 \pm 2^{\circ} \mathrm{C}, 50 \pm 10 \%$ relative humidity, and 12-h light/dark cycle. The acclimatization period was 5 days prior to the experiments. The present study was approved by The Scientific Review and Ethics Committee of Shandong Cancer Hospital and Institute (Jinan, China). The animals were shaved and the skin around the implantation sites was disinfected with $3 \%$ iodine and $75 \%$ alcohol prior to injection (19). Injection of the mixture was performed according to previously described procedures, with some modifications; increased injection depth in case of skin ischemia and injections were slow but withdrawn quickly to cause a hemispherical hump (4,20-23). The mice were anesthetized using a $80 \mathrm{mg} / \mathrm{kg}$ ketamine hydrochloride and $5 \mathrm{mg} / \mathrm{kg}$ xylazine hydrochloride intraperitoneal injection. The euthanasia of guinea pigs was conducted by intraperitoneal injection with sodium pentobarbital, $150 \mathrm{mg} / \mathrm{kg}$. The rabbits were euthanized with intravenous administered overdose of sodium pentobarbital
(100 mg/kg). Total anesthetic depth was defined as loss of response to the irritation of toes, cornea and skin and the loss of righting reflex. The experimental dilutions were injected into the subcutaneous tissues of dorsal skin between the upper limbs forming a round bulge at a $45^{\circ}$ angle using $1 \mathrm{ml}$ syringes. The mice were euthanized when the humane endpoints were observed or markers were no longer detected by CT imaging or palpation, using an overdose of ketamine $(240 \mathrm{mg} / \mathrm{kg})$ and xylazine $(20 \mathrm{mg} / \mathrm{kg})$ injected into the lateral tail vein. Death confirmation depended on a combination of criteria, including lack of pulse, breathing, corneal reflex and response to firm toe pinch, inability to hear respiratory and touch of heartbeat for more than $30 \mathrm{sec}$ with a stethoscope (24). Animals with any observable acute signs of pain and distress, such as increased scratching, poorer coat condition, weight loss, and a growing abdomen, which indicate a continuing and possibly irreversible deterioration in an animal's condition should be removed from the procedure and receive the appropriate treatment.

Experimental design. The study was designed in 3 parts: Macroscopic evaluation (part 1), microscopic evaluation (part 2) and biocompatibility evaluation (part 3) (Fig. 1). The macroscopic evaluations included analysis of systematic and local toxicity of materials and operation. The skin status and individual weight were routinely monitored weekly at certain points over a period of 7 weeks. Part 2 was conducted to examine the histopathological changes using hematoxylin and eosin (H\&E) staining and immunohistochemical analysis. All the measurements (parts 1: systemic side reactions, skin status scores, body weights, permanence of marker, quality of imaging; part 2: histopathological changes, inflammatory cells) were calculated and compared to determine the optimal NBCA/Lip combination. Then the biocompatibility of the chosen combination was investigated in part 3 using L929 mouse fibroblasts (Kunming Cell Bank of Type Culture Collection).

Macroscopic evaluations. There were four treatment groups with two mixing ratios (1:1 and 1:3) and two injection volumes (0.1 and $0.15 \mathrm{ml})$ as follows: 1:1-0.1, 1:1-0.15, 1:3-0.1 and 1:3-0.15 $\mathrm{ml}$. The ratios and dilutions used in the present study were based on pre-experiments, clinical experience and literature review (14,15,25-27). Sixty male Balb/c mice were equally and randomly divided into four treatment groups and a control group. The four treatment groups received different dilutions of NBCA/Lip and the control group received the same total volume $(0.15 \mathrm{ml})$ of saline solution to allow comparisons of weight increase with the treatment groups. Sample size required in previous studies was usually 6-12 mice for four groups with the two-sided significant level of 0.05 and a power of $90 \%$, however the present study was a type of multiple factorial exploration and a high power and effect size was needed for the credibility of the study (28-32). In addition, in the preliminary experiment, two mice died, which may have been caused by the blood vessel injections (14), therefore, the heterogeneity of variance could be decreased by 12 mice/group. Baseline data were acquired immediately following implantation and the skin scores, body weights and CT image scans were examined weekly over a period of 7 weeks. Follow-up CT scans were conducted 


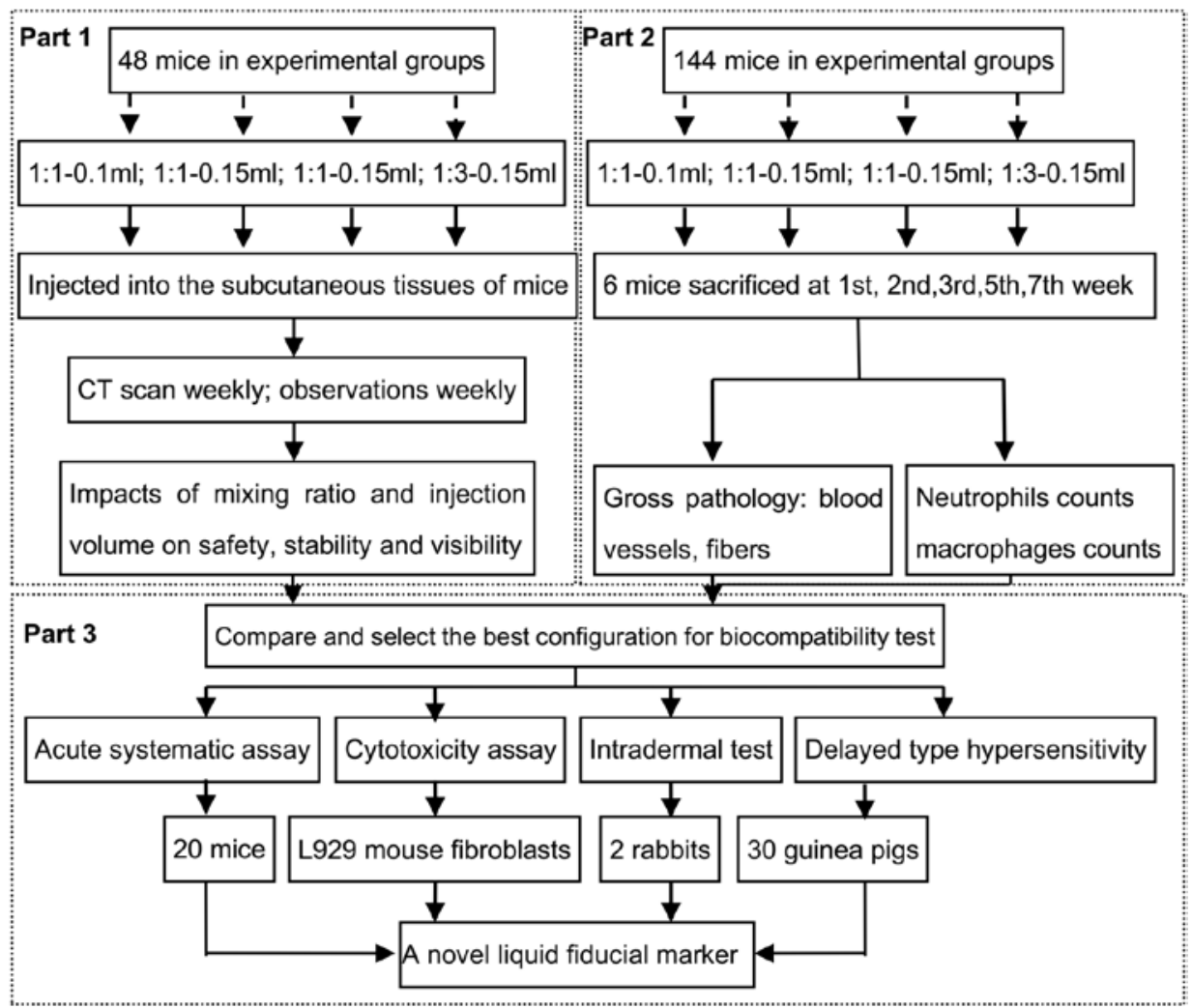

Figure 1. Experimental design chart. Part 1 shows the macroscopic evaluation; part 2 shows the microscopic evaluation; part 3 shows the biocompatibility evaluation.

monthly until 6 months or when the mixtures could no longer be detected by CT imaging or palpation. The initial observations continued from the time immediately following implantation until the animals completely recovered from the anesthesia and every hour during the subsequent $12-\mathrm{h}$ period. Observable side effects included lethargy, tremors and spasms, a staggering gait, twitches, a scruffy coat and death. The skin scoring system includes the sum of erythema scores $(0$, no reaction; 1 , very mild; 2 , mild with clear erythema or minimal ulceration; 3 , medium erythema or mild ulceration; 4 , severe reaction and eschar formation) and swelling scores ( 0 , no reaction; 1 , minimal swelling; 2 , mild with clear edema no more than the local margin; 3 , medium; 4 , severe with $1 \mathrm{~cm}$ above the skin and more than the injection sites). The higher the score, the worse the skin status was (16).

Permanence of imaging. All injected markers were evaluated for remaining volume using a MicroCAT II system (Siemens Healthineers) and this was contoured using a Hounsfield Unit (HU) thresholding function with a lower limit of $300 \mathrm{HU}$ for defining the contour of the marker and no upper limit was defined. Subsequent analysis was performed using Imaging Biomarker Explorer Software version V1.0 $\beta$ (MD Anderson Cancer Center).

Microscopic examinations. In Part 2, 144 mice were randomly divided into 4 treatment groups $(n=36)$ and 6 randomly selected mice were sacrificed from each group for histopathological examinations conducted at weeks $1,2,3,5$ and 7 post-injection and the remaining mice were used for follow-up observation.
Histopathological analysis. The mice were directly fixed on the operating table after death and a necropsy was performed within 2 min after euthanasia. After hair removal, the mixture, together with covered skin and subcutaneous tissue, was separated from the back of the mice with a scalpel and scissors, and the skin tissue containing mixtures was fixed with $10 \%$ formalin at $4^{\circ} \mathrm{C}$ for $24 \mathrm{~h}$ and embedded in paraffin. The slices with 4-um thickness were subjected to H\&E staining, which included the interface between mixtures and the tissue, and were evaluated by a pathologist blinded to the group conditions, treatment and sampling time of the specimens under a light microscope at $\times 200$ magnification.

Immunohistochemistry. The presence of inflammatory cells for quantitative comparisons of reactions to the NBCA/Lip mixture wereassessed using immunohistochemistry.Following deparaffinization in xylene (Servicebio Technology Co., Ltd.), the sections were heated $\left(98^{\circ} \mathrm{C}\right)$ for antigen retrieval by microwaving for $10 \mathrm{~min}$ in citrate buffer $(\mathrm{pH} \mathrm{6.0)}$. The primary antibodies, LY6G and CD68 (cat. no. GB11229; Servicebio Technology Co., Ltd.) diluted at 1:800, were added to detect neutrophils and macrophages, respectively. The corresponding secondary antibody, HRP goat anti-rabbit IgG (cat. no. G23303; Servicebio Technology Co., Ltd.) diluted at 1:200, was added and 3,3-diaminobenzidine (DAB) was added as a chromogen. Hematoxylin (Servicebio Technology Co., Ltd.) was used as the counterstain at $15^{\circ} \mathrm{C}$ for 3 minutes. The sections were then dehydrated in graded (50-100\%) alcohols, followed by xylene for $5 \mathrm{~min}$ each, cover-slipped and visualized under a light microscope. Positive staining 

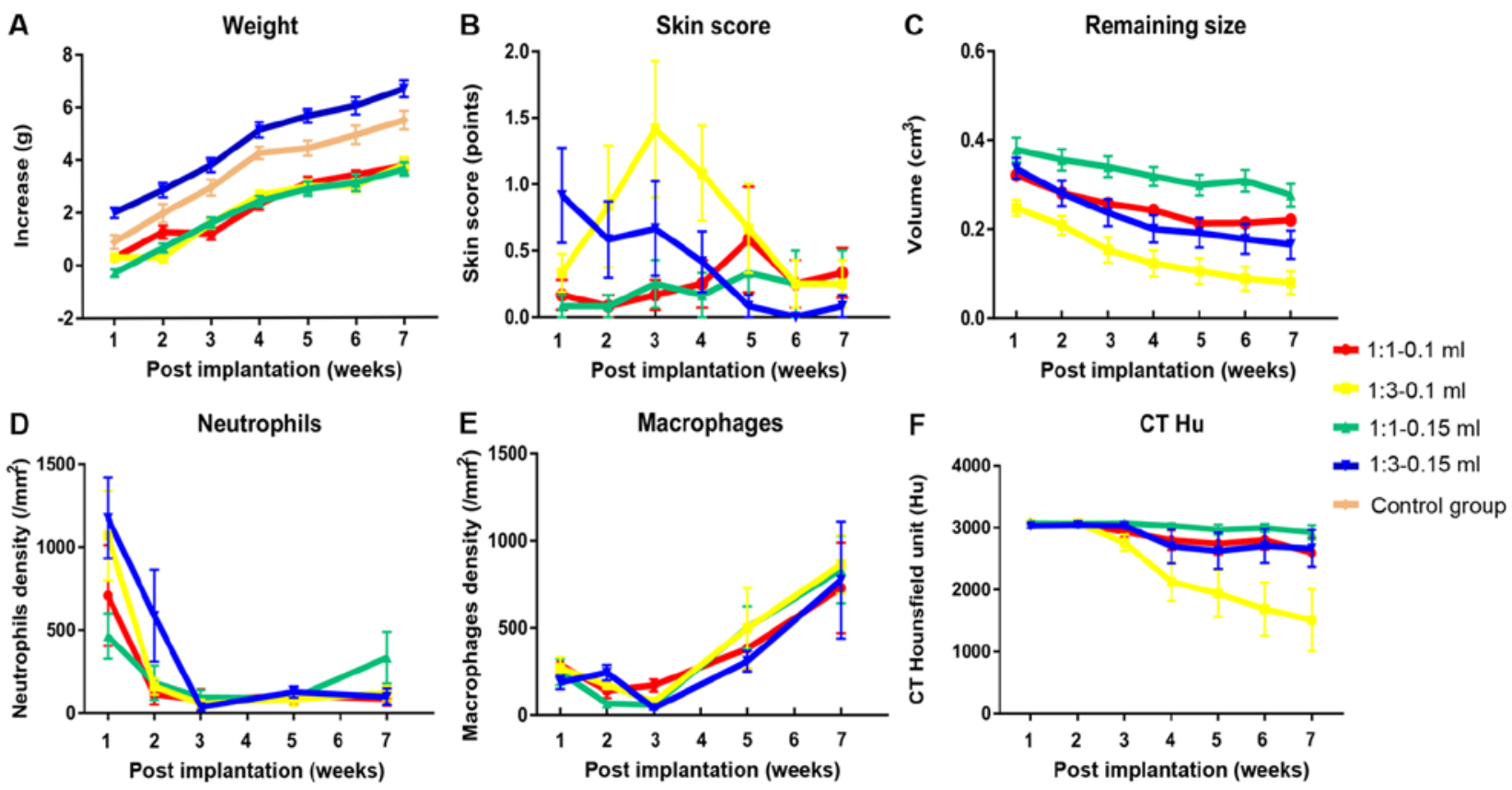

Figure 2. Measurements of each treatment group during the 7-week observation period. (A) Weight; (B) skin score; (C) remaining size of nodules; (D) neutrophil count; and (E) macrophage count; (F) CT HU. CT, computed tomography; HU, Hounsfield Unit.

was indicated by a brown color. A $5 \times 5 \mathrm{~mm}$-square grid was inserted into one of the ocular sections of a microscope. The images were captured under the microscope at a magnification of x200 and were analyzed using Image Pro Plus version 6.0 software (Media Cybernetics Inc.). At least 5 squares were positioned for each section.

Biocompatibility tests. The combination of 1:1-0.15 ml NBCA/Lip, which was chosen based on the comparison of the safety and effectiveness of different concentrations and proportions of the mixture in the first part and the second part, was subjected to biocompatibility evaluations, including four tests according to the ISO criteria. The conditioned media was extracted using $20 \mathrm{ml} 9 \%$ sodium chloride (SC) and $20 \mathrm{ml}$ cottonseed oil (CSO), respectively. An acute systematic assay was evaluated using 20 mice by intraperitoneal injection of 1:1-0.15 ml NBCA/Lip to examine the acute systematic toxicity according to ISO 10993.11 (17). The cytotoxic evaluation of the mixture was determined by performing an MTT assay using L929 mouse fibroblast according to ISO 10993.5 (18). L929 cells were cultured for $24 \mathrm{~h}$ at $37^{\circ} \mathrm{C}$ in 96 -well culture plates at an initial cell count of 10,000 cells/well and were subsequently exposed to the conditioned media (as aforementioned). Extraction solution from polyethylene was used as the negative control and the positive control was 10\% DMSO. The optical density of the supernatant was measured using a microplate reader (CrymeiBio, Ltd.) at $570 \mathrm{~nm}$. The delayed type hypersensitivity evaluation was ranked according to the Magnusson and Kligman scoring system in 30 Hartley Albino guinea pigs (Hua Fukang Bioscience, Ltd.), according to ISO 10993.10 (16). An intradermal test was administered with the samples injected in every 20 dorsal sites of two New Zealand white rabbits (Hua Fukang Bioscience, Ltd.), including 5 sites each for the SC treatment group, SC control group, CSO treatment group and the CSO control group. Skin scores were observed at 24,48 and $72 \mathrm{~h}$ post implantation, according to ISO 10993.10 (16).

Statistical analysis. All statistical analyses were performed using SPSS version 22.0 (IBM Corp) and the data are expressed as mean \pm standard error of the mean (unless otherwise shown). The two-way and three-way mixed ANOVAs were performed to examine the effects of factors of mixing ratio, injection volume and time and the differences between the four settings. The variables of mixing ratio and injection volume were treated as between-subject factors. Tests of within-subjects post-hoc simple effects were performed using Bonferroni's correction. $\mathrm{P}<0.05$ was considered to indicate a statistically significant difference.

\section{Results}

Macroscopic evaluations. Within-subject effects and between-subject effects are listed in Tables SI and SII. The results of simple effects and the main effect between mixing ratio and injection volume are listed in Table SIII and those involving the factor of time are listed in Table SIV.

No limitation of movement or change in behavior was observed. Two mice may have died of accidental intravenous injection of mixtures in the preliminary experiment after autopsy. Based on this knowledge, in the experiment, no death occurred and none of the mice had a severe infection or operation-related complications in the immediate and follow-up observations.

Weight changes of mice in part 1. Statistical analysis revealed that when the mixing ratio was $1: 1$, the injection volume had no significant effect on the weight; however, when the ratio was $1: 3$, the injection volume of $0.1 \mathrm{ml}$ induced a significant reduction in weight of $1.976 \mathrm{~g}$ compared with the volume of 
$0.15 \mathrm{ml}(\mathrm{P}<0.001$; Table SIII). Further analyses also revealed that when the injection volume was $0.1 \mathrm{ml}$, the mixing ratio had no significant effect; however, the mixing ratio of 1:1 was associated with a significant $2.084 \mathrm{~g}$ weight loss compared with the mixing ratio of $1: 3$ at the injection volume of $0.15 \mathrm{ml}$ $(\mathrm{P}<0.001$; Table SIII). At each time point, the injection volume of $0.1 \mathrm{ml}$ significantly decreased weight compared with the volume of $0.15 \mathrm{ml}(\mathrm{P}<0.001)$, excluding the 2 nd week $(\mathrm{P}=0.104)$ (Table SIV). In multiple comparisons, apart from the 1:3-0.15 ml group which was significantly larger compared with the other three settings, the average weight of the other three settings was significantly lower compared with the control group (1:1-0.1 ml, 22.09 $\pm 0.19 \mathrm{~g} ; 1: 1-0.15 \mathrm{ml}, 22.00 \pm 0.21 \mathrm{~g}$; 1:3-0.1 ml, $22.10 \pm 0.21 \mathrm{~g} ; 1: 3-0.15 \mathrm{ml}, 24.17 \pm 0.26 \mathrm{~g}$; control group, 23.37 $\pm 0.27 \mathrm{~g}$; Table I and Fig. 2A).

Skin status score of mice in part 1. For skin status, the effect of different ratios on skin score was dependent on time. It was observed that before the 4th week, the ratio of 1:1 was significantly associated with a more favorable skin status and after that the ratio of 1:1 was associated with a worse skin status, although this difference was not significant (Table SIV). Injection volume had no significant effect on skin status (Table SIII). The overall worst skin score was observed in the 1:1-0.15 ml group and the best skin score was associated with the 1:1-0.1 ml group; however, multiple comparisons revealed that this difference was not significant (1:1-0.1 ml, 0.27.71; $1: 1-0.15 \mathrm{ml}, 0.70 \pm .20 ; 1: 3-0.1 \mathrm{ml}, 0.69 \pm 1.19 ; 1: 3-0.15 \mathrm{ml}$,

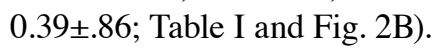

Permanence of imaging effects of markers in part 1. After 7 weeks of follow-up, NBCA/Lip injection nodules were observed in a total of 42 mice using CT images between the four treatment groups $(1: 1-0.1 \mathrm{ml}, 11 / 12 ; 1: 1-0.15 \mathrm{ml}, 12 / 12$; $1: 3-0.1 \mathrm{ml}, 8 / 12 ; 1: 3-0.15 \mathrm{ml}, 11 / 12)$. No obvious artifacts were observed using CT imaging. For the 1:3-0.1 ml combination, $33.3 \%$ of nodules could not be analyzed, indicating the most rapid degradation rate of the treatment mixture. The 1:3-0.15 ml group maintained a stable nodule for 7 weeks and fig. 3 shows a series of images of a nodule (Arrows in Fig. 3) in this treatment at 1, 3, 5, 7 weeks post implantation, which was not observed on the follow-up images at 3 months. Further analysis on the association between time and the mixing ratio showed that over the 7 weeks, the mixing ratio of $1: 1$ created a larger nodule compared with $1: 3(\mathrm{P}<0.001$, Table SIV) and the injection volume $0.1 \mathrm{ml}$ was $0.079 \mathrm{~cm}^{3}$ larger than the injection volume of $0.15 \mathrm{ml}(\mathrm{P}=0.001$, Table SIII). Multiple comparisons revealed significant differences between the 1:1-0.1 ml group vs. 1:3-0.1 ml group, 1:1-0.15 ml group vs. 1:3-0.1 ml group, 1:1-0.15 ml group vs. 1:3-0.15 ml group (Table I and Fig. 2C).

Dynamic changes of CT Hounsfield Units (HU). CT values are expressed in HU and were obtained by drawing the volumetric region of interest. The contrasted results between the different groups varied according to the post-treatment weeks. From the 4th week, the difference between the 1:1-0.15 and 1:3-0.1 ml groups became significant until the 7 th week Significant differences were also observed between the 1:1-0.1 and 1:1-0.15 $\mathrm{ml}$ groups at the 6th and 7 th week. For the 1:3-0.1 and 1:3-0.15 $\mathrm{ml}$ groups, the significant time point was the 7th week. Generally, at 7 weeks, the CTHU in the 1:3-0.1 ml group declined the most rapidly and the CTHU in 1:1-0.15 ml group kept stable change (Fig. 2F).

Histopathological analysis of markers in mice in part 2. Overall, the histopathology observed as a result of the different NBCA/Lip mixtures included inflammation, tissue granulation, minimal foreign body reactions and varying levels of fibrous capsule formation (Figs. 4 and 5). In the acute stage at 2 weeks, granulation was observed alongside focal infiltration of neutrophils as well as mild edema and dilated capillaries in all four treatment groups (Fig. 4A-D). However, a more intense reaction could be distinguished at the ratio of 1:1 (Fig. 4A and $\mathrm{B}$ ) compared with the ratio of 1:3 (Fig. 4C and D). Chronic granulomatous vasculitis and fibrosis of the nodules followed in the chronic stage (Fig. 5). At the 3rd week, chronic inflammation initiated an increase of fibroblast infiltration into the granulated tissue with local vascularization in all 4 groups, which reached a peak at the 5th week. By the 7th week, a fibrous capsule was marked with fibroblasts and multinuclear giant cells embracing particles in groups treated with the ratio of 1:1 (Fig. 5A and B). However, the premature tissue granulation with less intense fibroblast proliferation and fewer foreign giant cells were primarily observed in the groups treated with the ratio of $1: 3$ (Fig. 5C and D). Regardless of the different treatment combinations, the general appearance of the nodules ressemble that of a hard nodule until the 5th week; subsequently, the nodules treated with the 1:3 ratio were palpated softer compared with those treated with the ratio of 1:1. At the 3rd month, no nodule was palpated and visible on the CT images in the groups treated with the ratio of 1:3 and at 6 months for groups treated with 1:1.

Neutrophil counts of tissue in mice in part 2. LY6G-positve cells were quantified. For interactive effects, a significant difference was only observed between time and mixing ratio, ( $\mathrm{Df}=1.741 ; \mathrm{F}=3.626 ; \mathrm{P}=0.043$; Table SI). Simple effects analysis revealed that the ratio of 1:1 was associated with fewer neutrophils compared with the ratio of 1:3 at the 1 st week and no differences were observed at other time points (Table SIV). When the mixing ratio was 1:3, compared with the 1st week, the number of neutrophils significantly decreased gradually over time ( $\mathrm{P}=0.042$; Table SIV). Injection volume did not have a significant effect on the neutrophils counts (Table SIII). Finally, the differences between the four groups were examined and no significant differences were observed (Table I and Fig. 2D).

Macrophage counts of tissue in mice in part 2. Statistical tests of model effects on macrophage counts revealed no significant associations between cell counts and injection volume and injection ratio (Tables SI and SII). The analysis of the main effects demonstrated that compared with the 1st week, macrophage counts decreased significantly before the 3rd week and subsequently increased significantly in all groups (Table SIV). As for the four settings, no significant difference in macrophage counts was observed between post-implantation week 1 and 7 (Table I and Fig. 2E).

Biocompatibility tests in part 3. Based on the results of part 1 and part 2, the combination of 1:3-0.15 $\mathrm{ml}$ was selected to 


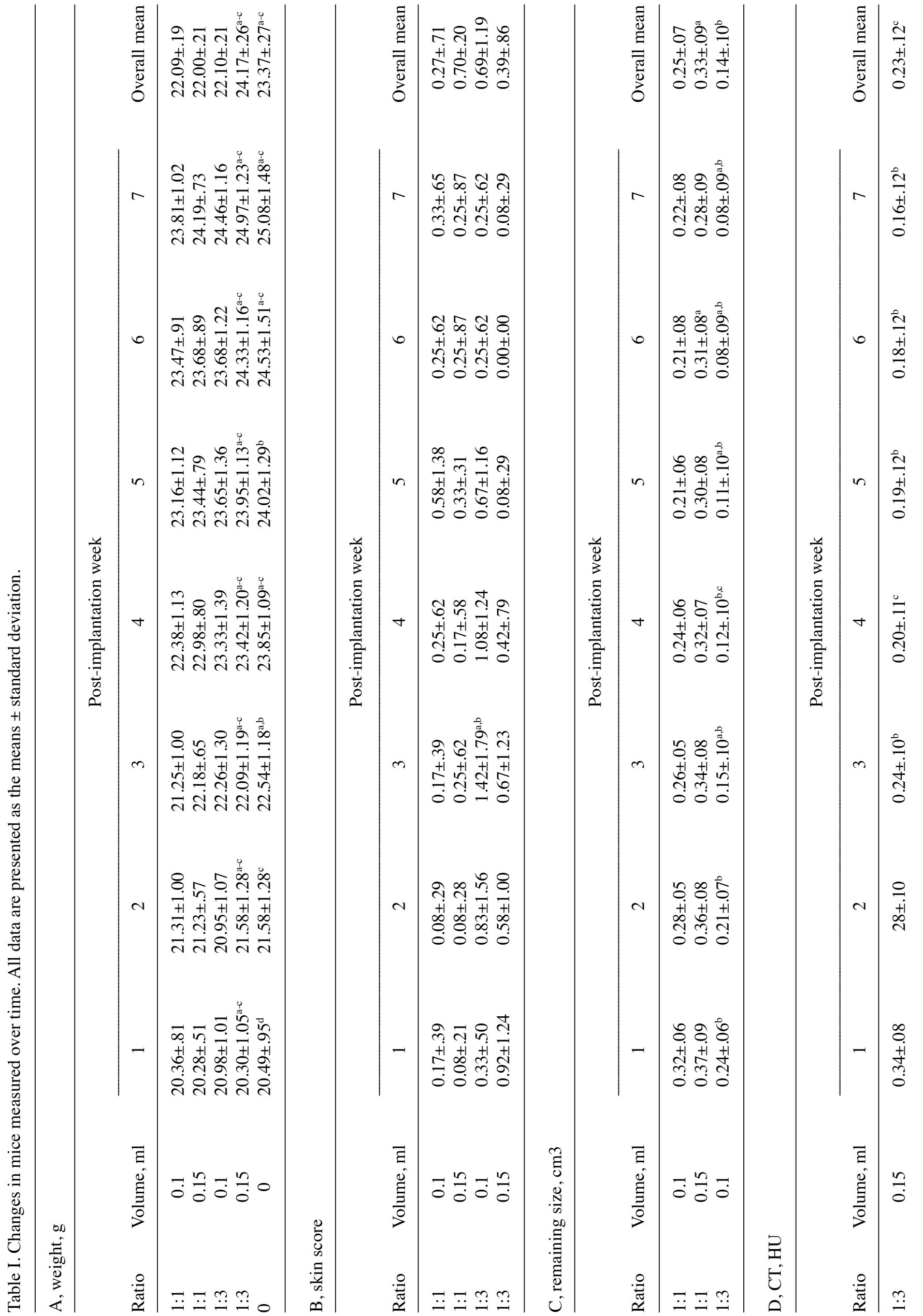



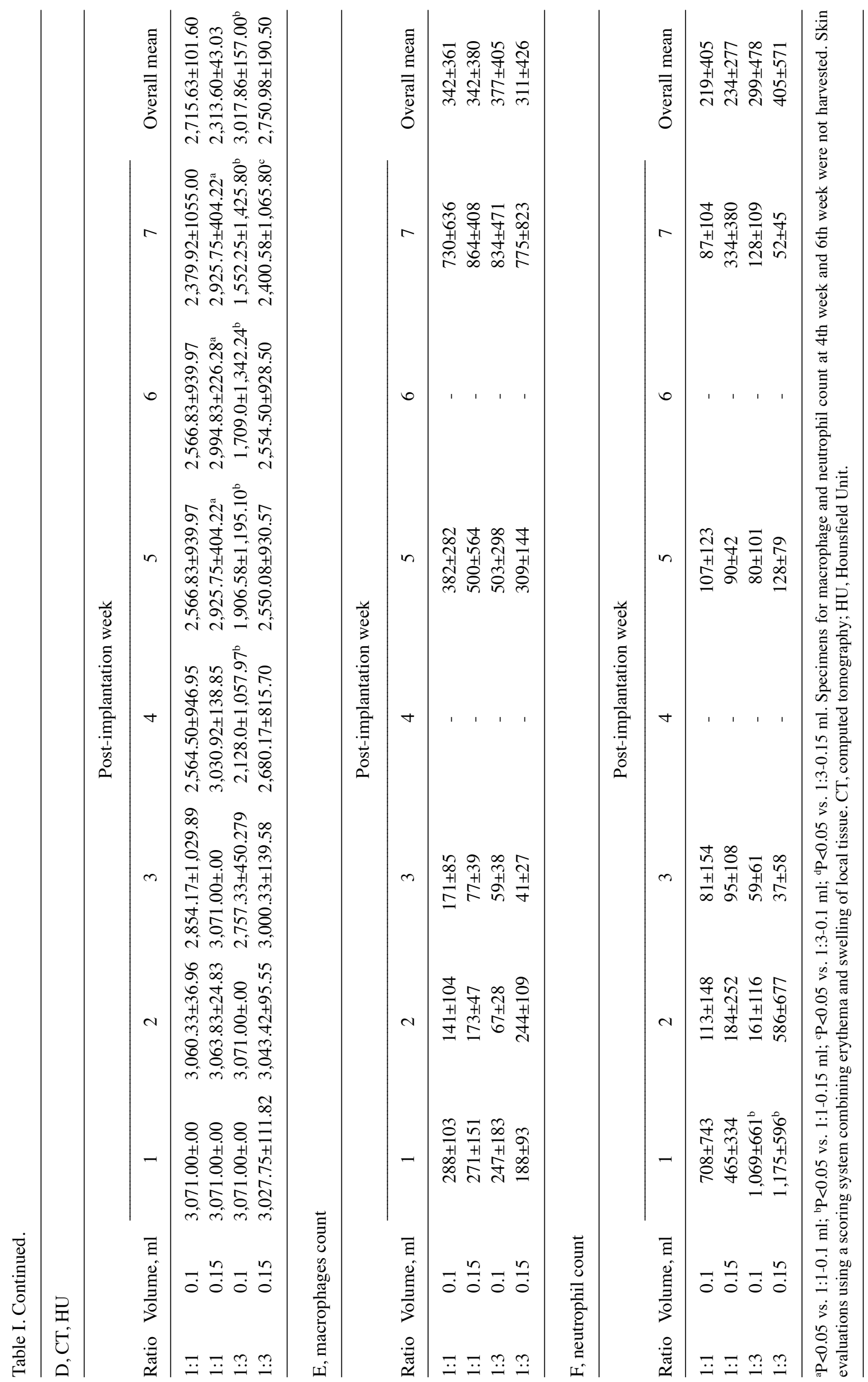

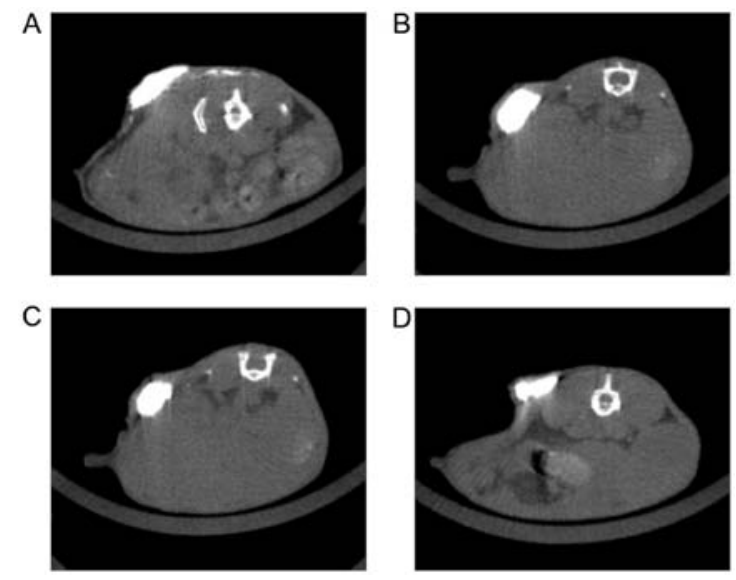

Figure 3. Nodules on CT images in mouse of 1:3-0.15 $\mathrm{ml}$ at different time points. (A) 1, (B) 3, (C) 5 and (D) 7 weeks post implantation. Arrows indicate the nodules of markers in subcutaneous tissue of mice in part 1.

further analyze biocompatibility, following the ISO 10993 criteria. The results of acute systematic assay showed that within the 72-h evaluations, no death occurred in the treatment or control group and the weight of mice increased gradually, irrespective of the extraction liquid (Fig. SI). The results of the MTT assay reported that the relative proliferation rate of the treatment group was $95 \%$ at the grade I level of cytotoxicity (Table SV). According to the Magnusson and Kligman classification criteria (16), the absence of distinct erythema and edema on guinea pigs' skin demonstrated a reaction grade $<1$ and all the guinea pigs exhibited no skin allergies in the treatment group. No difference was greater than 1 at 24, 48 and $72 \mathrm{~h}$ post-injection, which meant that either the SC or CSO extraction for the intradermal test were safe on rabbits within the 72-h evaluation (Table SVI).

\section{Discussion}

Fiducial placement is well established in breast and prostate cancer $(33,34)$ and the previous literature has suggested its value in radiation therapy for upper gastrointestinal tumors $(2,5,8,9)$. In the present study, the effects of mixing ratio and injection volume were evaluated and it was demonstrated that the higher concentration of NBCA (1:1) was associated with a more intense tissue response, resulting in a larger remaining volume and a longer degradation time compared with the lower mixing ratio (1:3). Nodules formed by a larger injection volume $(0.15 \mathrm{ml})$ were associated with a larger remaining volume as observed using CT imaging. The combination of 1:3-0.15 ml injection into subcutaneous tissue was associated with improved fusion with local tissue and this combination maintained a more stable imaging nodule in CT images during the initial 7 weeks. The final biocompatibility test demonstrated the safety of $1: 3-0.15 \mathrm{ml}$ NBCA/Lip according to the ISO criteria. Therefore, this ratio should be further investigated in experiments involving larger animals.

The two ratios and two volumes used in the present study were selected based on pre-experimental data. A high viscosity and long polymerization time of fiducial markers, which increases proportionately with decreased NBCA volume, will impede the operation and limit the precision of marking the site $(14,15,25)$. In a study conducted by Stoesslein et al (26), when the NBCA concentration increased from 20 to $25 \%$, this yielded an increase in polymerization time from $7.5 \pm 0.8$ to $11.8 \pm 1.5 \mathrm{sec}(26)$. In addition, in the preliminary experiment on BALB/c mice, NBCA was diluted over 1:3 resulted in various flat shapes easily squeezed by surrounding tissues, and when injecting volumes was $0.1-0.2 \mathrm{ml}$, a nodule $\sim 1 \mathrm{~cm}$ in diameter on the CT images was created without significant leakage, which was in accordance with a previous study (27).

The present evaluations of the reactions to different mixture compositions of NBCA/Lip demonstrated that the 1:3-0.15 ml combination was the safest. Body weight and skin status at the injection site were individually monitored as these were expected to be sensitive indicators of adverse reactions associated with the implantation $(35,36)$. Although the results did not indicate growth retardation as a result of implantation, it was observed that the combination of 1:3-0.15 ml had the least impact on animal weight compared with the 3 other mixtures. Notably, with the configurations of 1:1-0.15 and 1:3-0.1 ml, significantly greater extents of erythema and edema were observed. Schineider and Otto (37) reported that the reaction degree of soft tissue to Histoacryl ${ }^{\circledR}$ was associated with the mixture volumes and ratios in vitro and in vivo, as further supported by the present study. The present study reported that with greater volumes of NBCA, the extent of the reaction was greater and the degradation rate was considerably attenuated. Inflammatory reactions can occur in chemically or physically injured tissues $(2,9,38)$. However, the minimal dosage and appropriate configuration of NBCA/Lip limited the toxicity observed in the present study.

The biodegradation of an implant in vivo depends on a number of factors, including the microscopic appearance of the implantation, the interaction of tissue-interface and the histopathological process $(2,39)$. When NBCA/Lip is treated as permanent embolic material, the microscopic appearance of the solidified mass after blocking blood vessels has been reported to be a honeycombed lattice containing blood clots in the channel, which may become organized and recanalized (40-42). NBCA adheres to the tissue, but lipiodol reduces this capacity and causes peripheral solidification, which notably affects the microscopic tissue interface. Generally, the low concentration and a small volume of NBCA/Lip may raise concerns regarding the remaining time of the radio-opaque mass on radiographs. For example, in the present study the 1:3-0.1 ml combination resulted in the smallest number of markers visible on CT images at the 7th week. In addition, the skin score of 1:3-0.1 ml increased after injection, reached its peak during the 3rd week, and subsequently decreased gradually, returning to the mean value at 6th week. One explanation for this could be that this combination degraded faster, in accordance with the present examinations of imaging volume that the setting of 1:3-0.1 $\mathrm{ml}$ had only 8 nodules on images at 7 weeks and the lowest CT HU among four configurations. Low density of NBCA and low injection volume may create a looser structure, resulting in a faster degradation and affect the skin reaction at early stage. Moreover, it was hypothesized that when the density of NBCA is low, the polymerization time is longer, which results in lower catheter occlusion and more distal penetration into small vessels, further irritating the skin. This 

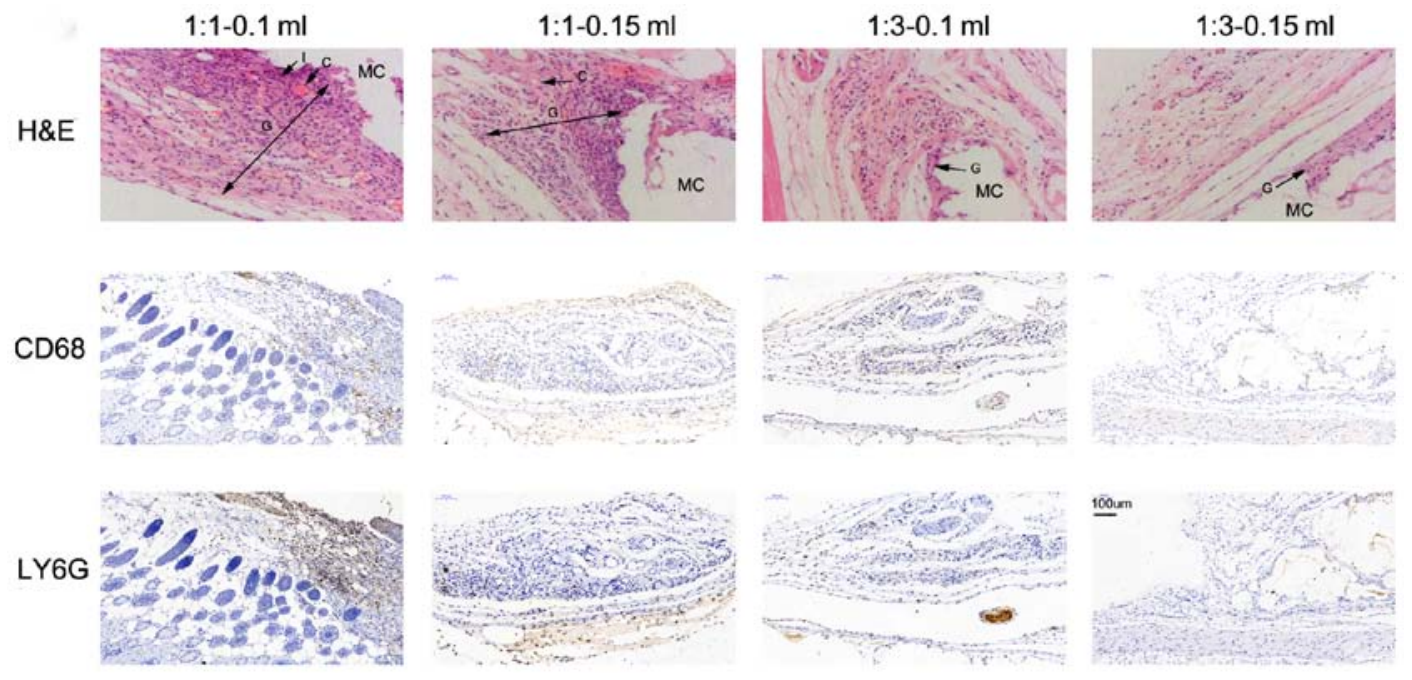

Figure 4. H\&E and immunohistochemical images (CD68 and LY6G) of N-butyl cyanoacrylate/lipiodol implantation into subcutaneous tissue during the acute inflammatory phase at 2 weeks post implantation from mice in part 2 in four treatment groups. Massive granulation tissue formation with inflammatory cell infiltration surrounding the material treated with 1:1 (A and B). Scattered granulation tissue around the embedded material treated with 1:3 (C and D). H\&E, the first row. CD68-positive cells indicate macrophages (brown), the second row. LY6G-positive cells indicate neutrophils (brown), the third row. $\mathrm{H} \& \mathrm{E}$, hematoxylin and eosin staining; I, inflammatory cells; C, capillary; G, granulation tissue; MC, material cavity.
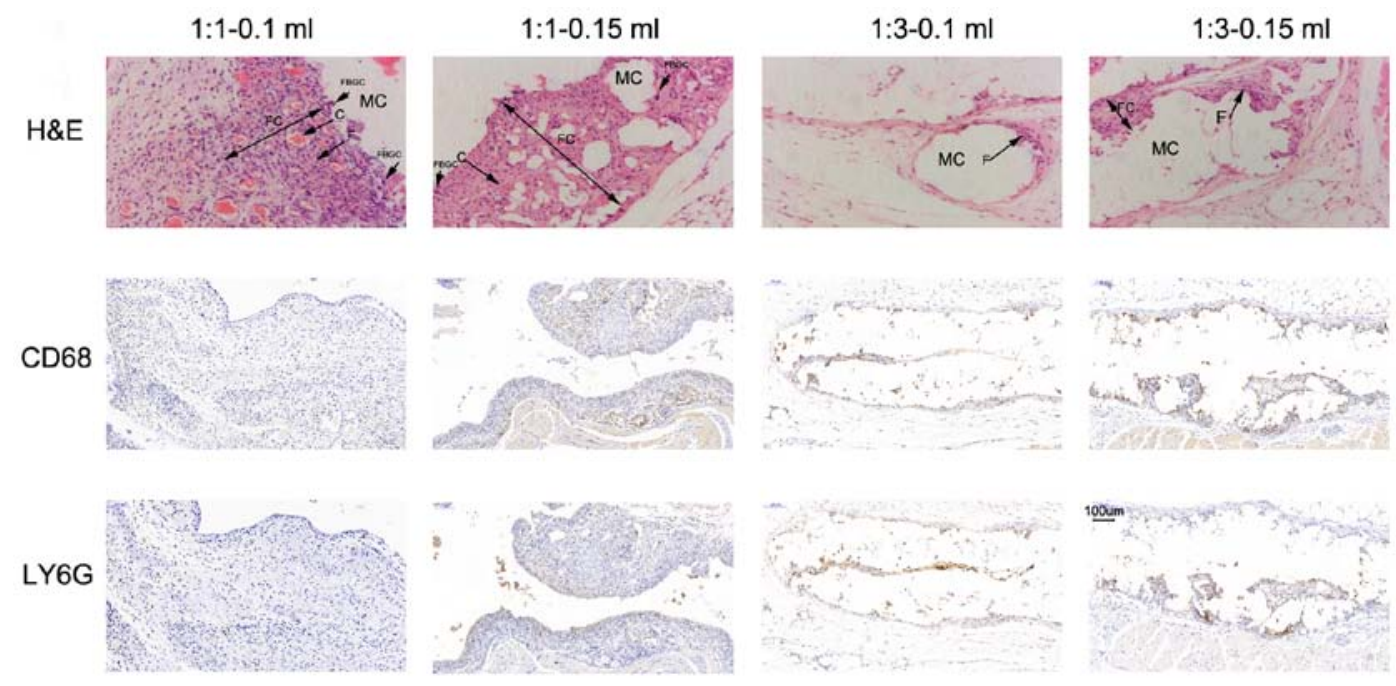

Figure 5. H\&E and immunohistochemical (CD68 and LY6G) images of N-butyl cyanoacrylate and lipiodol implantation in subcutaneous tissue during the chronic inflammatory phase at 7 weeks from mice in part 2 in four treatment groups. Fibrous capsules from granulation tissue at the tissue repair phase. Thick layered fibrous capsule with massive macrophages surrounding the materials treated with 1:1-0.1 ml (A) and 1:1-0.15 ml (B). Thin layered fibrous capsule tissue around the material in low ratio of 1:3-0.1 $\mathrm{ml}(\mathrm{C})$ and 1:3-0.15 $\mathrm{ml}$ (D). Seldom neutrophils were observed at the tissue repair phase. CD68-positive cells indicate macrophages (brown). LY6G-positive cells indicate neutrophils (brown). H\&E, hematoxylin and eosin staining; G, granulation tissue; MC, material cavity; C, capillary; I, inflammatory cells; FC, fibrous capsule; F, fibroblasts; FBGC, foreign body giant cell. Magnification, x200.

hypothesis is supported by previous studies investigating embolization treatment (43-47). In the present study, increasing the injection volume or the concentration of NBCA created a larger remaining size of the nodule over time. The worse skin status in treatment of 1:3-0.1 ml may be partially explained by the low concentration of NBCA in NBCA/Lip. Rydhog et al (23) estimated the relative volume change of a liquid fiducial marker for radiotherapy in patients with lung cancer and at end of radiotherapy treatment was $-23 \%$ for tumor injections and $-5 \%$ for lymph node injected markers. Thick fibrosis and decreased blood flow, as a result of the increased extracellular matrix and reduced blood circulation, are consequently considered to be involved in the persistence of the CT imaging effect.
Another factor in the measurement of the structural stability of fiducial markers is HU variation, which can impact the ability to consistently measure the marker volume (23). As for the radio-opacity (HU), Rydhog et al (23) reported notably lower radio-opacity of markers injected into the lymph node compared with those injected into the tumors. The authors attributed this to the degree of tissue condensing following injection, wherein tissue volumetric integrity was better preserved and an improved representation of the tumor border was observed. Other studies have demonstrated that a large volume of liquid material leads to hardened artifacts and results in decreased imaging quality, rendering the radiotherapy unprecise $(12,48)$. In the present study, the injection volume of $0.15 \mathrm{ml}$ was not 
shown to reduce the CT imaging quality. Overall, it is valuable that the application of 1:3-0.15 $\mathrm{ml}$ created clear visible nodules for 7 weeks, which then rapidly diminished, meeting the four criteria of fiducial markers as outlined by Habermehl et al (7), including the visibility, the absence of artifacts, easy application and sufficient immovability.

Following an evaluation of safety and stability of different combinations of NBCA/Lip, the 1:3-0.15 $\mathrm{ml}$ setting is recommended for the use of this fiducial mixture in further research. Given that no previous data have illustrated the biocompatibility of this specified setting and biocompatibility is a prerequisite for use in human patients, the present toxicity evaluation was conducted following the ISO criteria and the results revealed that this material has a good biocompatibility.

Cyanoacrylates are hypothesized to be cytotoxic primarily at the initial dehydration stage of the tissues before full polymerization and are then removed slowly by macrophages $(15,21,37,38,49,50)$. The release of formaldehyde, a compound produced in the process of the polymerization of cyanoacrylates, which releases fewer residues during cell culture and, therefore, should be less cytotoxic, was demonstrated to be less cytotoxic compared with cyanoacrylates in a previous study (49). Data regarding the biocompatibility of NBCA and Lip are rare; however, the histopathological examinations of embolization in veins and arteries of different parts of the body are within the acceptable range $(26,39,41,51)$. Furthermore, the dosage used in the present study was less than that used in interventional embolization, contributing to the good biocompatibility of NBCA and Lip as fiducial markers.

Clinically, fiducial markers should be injected close to the tumor margins, which would likely influence the histopathology and degradation rate $(12,21-23)$. Therefore, it is difficult to determine whether the histological changes and the permanent effects are due to the underlying disease. Moreover, the tissue reactions and degradation rates reported in the present study demonstrate that the extrapolation of the different mixing ratios and volumes to a wider range should be approached with caution. Accordingly, these results highlight the need for further assessment of the NBCA/Lip mixture quantities prior to the use of this mixture in human patients.

In conclusion, the combination of 1:3-0.15 ml NBCA/Lip is a good fiducial marker in vivo which may have valuable applications in IGRT, as these mixture quantities were associated with limited granulomatous reactions, creating a well-balanced integration with tissue environment and, importantly, maintaining a stable nodule size and density for 7 weeks post implantation. Evaluations following ISO also showed the good biocompatibility of this setting. Overall, NBCA/Lip with the appropriate combination is a promising fiducial marker for IGRT and should be further investigated in other preclinical models.

\section{Acknowledgements}

Not applicable.

\section{Funding}

This work was supported by National Natural Science Foundation of China (grant no. 81972864), Science and
Technology Support Plan for Youth Innovation Teams of Universities in Shandong Province (grant no. 2019KJL001) and Science and Technology Plan of Jinan (grant no. 201907113).

\section{Availability of data and materials}

The datasets used and/or analyzed during the current study are available from the corresponding author on reasonable request.

\section{Authors' contributions}

LS, YS, CL, LW, XS and WL conducted the experiments and LS wrote the manuscript. XD and DX conducted the acquisition of data, data analysis and interpretation of data. JY and XM designed the study. All authors read and approved the final manuscript.

\section{Ethics approval and consent to participate}

Animal protocols were conducted in compliance with the regulations of the Shandong Cancer Hospital and the present study as approved by The Scientific Review and Ethics Committee of Shandong Cancer Hospital and Institute (Jinan, China; approval no. SDTHEC-2019030915).

\section{Patient consent for publication}

Not applicable.

\section{Competing interests}

The authors declare that they have no competing interests.

\section{References}

1. Welsh J, Settle SH, Amini A, Xiao L, Suzuki A, Hayashi Y, Hofstetter W, Komaki R, Liao Z and Ajani JA: Failure patterns in patients with esophageal cancer treated with definitive chemoradiation. Cancer 118: 2632-2640,2012.

2. Jin P, Van Der Horst A, De Jong R, van Hooft JE, Kamphuis M, van Wieringen N, Machiels M, Bel A, Hulshof MC and Alderliesten T: Marker-based quantification of interfractional tumor position variation and the use of markers for setup verification in radiation therapy for esophageal cancer. Radiother Oncol 117: 412-418, 2015

3. Burmeister BH, Beukema J, Guidi R, Harvey JA, Gotley D and Smithers BM: Localization of small esophageal cancers for radiation planning using endoscopic contrast injection: Report on a series of eight cases. Dis Esophagus 14: 28-31, 2001.

4. Chavalitdhamrong D, Dimaio CJ, Siersema PD and Wagh MS: Technical advances in endoscopic ultrasound-guided fiducial placement for the treatment of pancreatic cancer. Endosc Int Open 3: E373-E377, 2015.

5. Machiels M, Van Hooft J, Jin P, van Berge Henegouwen MI, van Laarhoven HM, Alderliesten $\mathrm{T}$ and Hulshof MC: Endoscopy/EUS-guided fiducial marker placement in patients with esophageal cancer: A comparative analysis of 3 types of markers. Gastrointest Endosc 82: 641-649, 2015.

6. Shirato H,Harada T, Harabayashi T, Hida K, Endo H, Kitamura K, Onimaru R, Yamazaki K, Kurauchi N, Shimizu T, et al: Feasibility of insertion/implantation of 2.0-mm-diameter gold internal fiducial markers for precise setup and real-time tumor tracking in radiotherapy. Int J Radiat Oncol Biol Phys 56: 240-247, 2003.

7. Habermehl D, Henkner K, Ecker S, Jakel O, Debus J and Combs SE: Evaluation of different fiducial markers for image-guided radiotherapy and particle therapy. J Radiat Res 54 (Suppl 1): i61-i68, 2013. 
8. Fukada J, Hanada T, Kawaguchi O, Ohashi T, Takeuchi H, Kitagawa Y, Seki S, Shiraishi Y, Ogata H and Shigematsu N: Detection of esophageal fiducial marker displacement during radiation therapy with a 2 -dimensional on-board imager: Analysis of internal margin for esophageal cancer. Int J Radiat Oncol Biol Phys 85: 991-998, 2013.

9. Fernandez DC, Hoffe SE, Barthel JS, Vignesh S, Klapman JB, Harris C, Almhanna K, Biagioli MC, Meredith KL, Feygelman V, et al: Stability of endoscopic ultrasound-guided fiducial marker placement for esophageal cancer target delineation and image-guided radiation therapy. Pract Radiat Oncol 3: 32-39, 2013.

10. Scherman Rydhog J, Perrin R, Jolck RI, Gagnon-Moisan F, Larsen KR, Clementsen P, Riisgaard de Blanck S, Fredberg Persson G, Weber DC, Lomax T, et al: Liquid fiducial marker applicability in proton therapy of locally advanced lung cancer. Radiother Oncol 122: 393-399, 2017.

11. Picardi C, Rouzaud M, Kountouri M, Lestrade L, Vallée JP, Caparrotti F, Dubouloz A, Miralbell R and Zilli T: Impact of hydrogel spacer injections on interfraction prostate motion during prostate cancer radiotherapy. Acta Oncol 55: 834-838, 2016.

12. Dobiasch S, Kampfer S, Burkhardt R, Schilling D, Schmid TE, Wilkens JJ and Combs SE: BioXmark for high-precision radiotherapy in an orthotopic pancreatic tumor mouse model: Experiences with a liquid fiducial marker. Strahlenther Onkol 193: 1039-1047, 2017.

13. Sondergaard J, Olsen KO, Muren LP, Elstrom UV, Grau C and Hoyer M: A study of image-guided radiotherapy of bladder cancer based on lipiodol injection in the bladder wall. Acta Oncol 49: 1109-1115, 2010.

14. Takeuchi Y, Morishita H, Sato Y, Hamaguchi S, Sakamoto N, Tokue H, Yonemitsu T, Murakami K, Fujiwara H, Sofue K, et al: Guidelines for the use of NBCA in vascular embolization devised by the Committee of Practice Guidelines of the Japanese Society of Interventional Radiology (CGJSIR), 2012 edition. Jpn J Radiol 32: 500-517, 2014.

15. Takasawa C, Seiji K, Matsunaga K, Matsuhashi T, Ohta M, Shida S, Takase K and Takahashi S: Properties of N-butyl cyanoacrylate-iodized oil mixtures for arterial embolization: In vitro and in vivo experiments. J Vasc Interv Radiol 23: 1215-1221.e1, 2012.

16. International Organization for Standardization, ISO 10993 10:2002. Biological evaluation of medical devices-Part 10: Tests for irritation and delayed-type hypersensitivity, 2002

17. International Organization for Standardization, ISO 10993-11:1993 Biological evaluation of medical devices-Part 11: Tests for systemic toxicity, 1993.

18. International Organization for Standardization, ISO 10993-5:1999 biological evaluation of medical devices-Part 5: Test for in vitro cytotoxicity, 1999.

19. Flecknell P, Lofgren JLS, Dysson MC, Marini RR, Swindle MM and Wilson RP: Chapter 24 - Preanesthesia, Anesthesia, Analgesia, and Euthanasia, In: Laboratory Animal Medicine. 3rd edition. American College of Laboratory Animal Medicine, p1135-1200, 2015.

20. Pos F, Bex A, Dees-Ribbers HM, Betgen A, Van Herk M and Remeijer P: Lipiodol injection for target volume delineation and image guidance during radiotherapy for bladder cancer Radiother Oncol 93: 364-367, 2009.

21. Rose M, Siva S, Ball D, Irving LB and Steinfort DP: Bronchoscopic delivery of lipiodol as a fiducial marker in lung tumors before radiotherapy. J Thorac Oncol 9: 1579-1583, 2014.

22. Freilich JM, Spiess PE, Biagioli MC, Fernandez DC, Shi EJ, Hunt DC, Gupta S and Wilder RB: Lipiodol as a fiducial marker for image-guided radiation therapy for bladder cancer. Int Braz J Urol 40: 190-197, 2014.

23. Rydhog JS, Mortensen SR, Larsen KR, Clementsen P, Jølck RI, Josipovic M, Aznar MC, Specht L, Andresen TL, Rosenschöld PM and Persson GF: Liquid fiducial marker performance during radiotherapy of locally advanced non-small cell lung cancer Radiother Oncol 121: 64-69, 2016.

24. Leary S, Underwood W, Anthony R et al: AVMA Guidelines for the Euthanasia of Animals: 2013 Edition. American Veterinary Medical Association, 2013.

25. Seewald S, Ang TL, Imazu H, Naga M, Omar S, Groth S Seitz U, Zhong Y, Thonke F and Soehendra N: A standardized injection technique and regimen ensures success and safety of $\mathrm{N}$-butyl-2-cyanoacrylate injection for the treatment of gastric fundal varices (with videos). Gastrointest Endosc 68: 447-454, 2008
26. Stoesslein F, Ditscherlein G and Romaniuk PA: Experimental studies on new liquid embolization mixtures (histoacryl-lipiodol, histoacryl-panthopaque). Cardiovasc Intervent Radiol 5: 264-267, 1982.

27. Kwon WJ, Kim HJ, Jeong YJ, Lee CH, Kim KI, Kim YD and Lee JH: Direct lipiodol injection used for a radio-opaque lung marker: stability and histopathologic effects. Exp Lung Res 37: 310-317, 2011

28. Chang Y, Qin J, Peng WD, Shi S, Fu SZ, Guo G, Hu HZ, Zhao X, Wei YQ and Qian ZY: Acute toxicity evaluation of biodegradable in situ gel-forming controlled drug delivery system based on thermosensitive PEG-PCL-PEG hydrogel. J Biomed Mater Res B Appl Biomater 91: 26-36, 2009.

29. Wu Y, Wang L, Zhang K, Zhou L, Zhang X, Jiang X and Zhu C: N-Butyl-2-cyanoacrylate-based injectable and in situ-forming implants for efficient intratumoral chemotherapy. Drug Deliv 24: 729-736, 2017.

30. Dalu A, Blaydes BS, Lomax LG and Delclos KB: A comparison of the infammatory response to a polydimethylsiloxane implant in male and female Balb/c mice. Biomaterials 21: 1947-1957, 2000.

31. Schmidt SAJ, Lo S and Hollestein LM: Research Techniques Made Simple: Sample size estimation and power calculation. J Invest Dermatol 138: 1678-1682, 2018.

32. Buch T, Moos K, Ferreira FM, Fröhlich H, Gebhard C and Tresch A: Benefits of a factorial design focusing on inclusion of female and male animals in one experiment. J Mol Med (Berl) 97: 871-877, 2019

33. Singh J, Greer PB, White MA, Parker J, Patterson J, Tang CI, Capp A, Wratten C and Denham JW: Treatment-related morbidity in prostate cancer: A comparison of 3-dimensional conformal radiation therapy with and without image guidance using implanted fiducial markers. Int J Radiat Oncol Biol Phys 85: 1018-1023, 2013.

34. Kirby AN, Jena R, Harris EJ, Evans PM, Crowley C, Gregory DL and Coles CE: Tumour bed delineation for partial breast/breast boost radiotherapy: What is the optimal number of implanted markers? Radiother Oncol 106: 231-235, 2013.

35. Vaisman B, Motiei M, Nyska A and Domb AJ: Biocompatibility and safety evaluation of a ricinoleic acid-based poly(esteranhydride) copolymer after implantation in rats. J Biomed Mater Res A 92: 419-431, 2010.

36. Miura C, Shimizu Y, Imai Y, Mukai T, Yamamoto A, Sano Y, Ikeo N, Isozaki S, Takahashi T, Oikawa M, et al: In vivo corrosion behaviour of magnesium alloy in association with surrounding tissue response in rats. Biomed Mater 11: 025001, 2016.

37. Schneider G and Otto K: In vitro and in vivo studies on the use of Histoacryl ${ }^{(\circledR)}$ as a soft tissue glue. Eur Arch Otorhinolaryngol 269: 1783-1789, 2012

38. Vinters HV, Galil KA, Lundie MJ and Kaufmann JC: The histotoxicity of cyanoacrylates. A selective review. Neuroradiology 27: 279-291, 1985.

39. Gounis MJ, Lieber BB, Wakhloo AK, Siekmann R and Hopkins LN: Effect of glacial acetic acid and ethiodized oil concentration on embolization with N-butyl 2-cyanoacrylate: An in vivo investigation. AJNR Am J Neuroradiol 23: 938-944, 2002.

40. Wikholm G: Occlusion of cerebral arteriovenous malformations with N-butyl cyano-acrylate is permanent. AJNR Am J Neuroradiol 16: 479-482, 1995 .

41. Sadato A, Wakhloo AK and Hopkins LN: Effects of a mixture of a low concentration of n-butylcyanoacrylate and ethiodol on tissue reactions and the permanence of arterial occlusion after embolization. Neurosurgery 47: 1195-1204, 2000.

42. Gruber A, Mazal PR, Bavinzski G, Killer M, Budka H and Richling B: Repermeation of partially embolized cerebral arteriovenous malformations: A clinical, radiologic, and histologic study. AJNR Am J Neuroradiol 17: 1323-1331, 1996.

43. Rafat M, Xeroudaki M, Koulikovska M, Sherrell P, Groth F, Fagerholm P and Lagali N: Composite core-and-skirt collagen hydrogels with differential degradation for corneal therapeutic applications. Biomaterials 83: 142-155, 2016.

44. Wilson DJ, Chenery DH, Bowring HK, Wilson K, Turner R, Maughan J, West PJ and Ansell CW: Physical and biological properties of a novel siloxane adhesive for soft tissue applications. J Biomater Sci Polym Ed 16: 449-472, 2005.

45. Brothers MF, Kaufmann JC, Fox AJ and Deveikis JP: n-Butyl2-cyanoacrylate-substitute for IBCA in interventional neurodiology: Histopathologic and polymerization time studies. AJNR Am J Neurodiol 10: 777-786, 1989. 
46. Pollak JS and White RI Jr: The use of cyanoacrylate adhesives in peripheral embolization. J Vasc Interv Radiol 12: 907-913, 2001.

47. Ishikawa M, Horikawa M, Yamagami T, Uchida BT, Awai K and Kaufman JA: Embolization of Arteriovenous Malformations: Effect of flow control and composition of n-Butyl-2 cyanoacrylate and iodized oil mixtures with and without ethanol in an in vitro model. Radiology 279: 910-916, 2016.

48. Lock M, Malayeri AA, Mian OY, Mayr NA, Herman JM and Lo SS: Computed tomography imaging assessment of postexternal beam radiation changes of the liver. Future Oncol 12: 2729-2739, 2016

49. Montanaro L, Arciola CR, Cenni E, Ciapetti G, Savioli F, Filippini F and Barsanti LA: Cytotoxicity, blood compatibility and antimicrobial activity of two cyanoacrylate glues for surgical use. Biomaterials 22: 59-66, 2001
50. Derks CM and Jacobovitz-Derks D: Embolic pneumopathy induced by oleic acid. A systematic morphologic study. Am J Pathol 87: 143-158, 1977.

51. Wang YM, Cheng LF and Li N: Histopathological study of vascular changes after intra-arterial and intravenous injection of N-butyl-2-cyanoacrylate. Chin J Dig Dis 7: 175-179, 2006.

This work is licensed under a Creative Commons Attribution-NonCommercial-NoDerivatives 4.0 International (CC BY-NC-ND 4.0) License. 